\title{
The suppressing effects of VEGF-mediated angiogenesis at different administration sequences of apatinib and transarterial embolization in vivo
}

\author{
Nan Li, Yong-Hui Huang \\ Department of Interventional Radiology, the First Affiliated Hospital, Sun Yat-sen University, Guangzhou 510080, China \\ Contributions: (I) Conception and design: YH Huang, N Li; (II) Administrative support: YH Huang; (III) Provision of study materials or patients: N \\ Li; (IV) Collection and assembly of data: N Li; (V) Data analysis and interpretation: YH Huang, N Li; (VI) Manuscript writing: All authors; (VII) \\ Final approval of manuscript: All authors. \\ Correspondence to: Dr. Yong-Hui Huang; Dr. Nan Li. Department of Interventional Radiology, the First Affiliated Hospital, Sun Yat-sen University, \\ 58th Zhongshan Road II, Guangzhou 510080, China. Email: cir.huangyonghui@vip.163.com; zsylinan@163.com.
}

Background: To investigate the suppressing effects of vascular endothelial growth factor (VEGF)-mediated angiogenesis at different treatment schedules by applying apatinib combined with transarterial embolization (TAE).

Methods: Forty ideal liver cancer rat models were randomly divided into four groups based on different administration methods of apatinib [control group (CG): TAE only; Combined Group 1 (CG1): apatinib administration 3 days pre-TAE; Combined Group 2 (CG2): apatinib administration simultaneously with TAE; Combined Group 3 (CG3): apatinib administration 3 days post-TAE]. The characteristics of liver cancer, the expression of VEGF and microvascular density (MVD) as determined by CD34, and the overall survival (OS) were compared among the groups.

Results: The tumor sizes of the liver on the 10th day after treatment were significantly larger when compared to the baseline sizes $(\mathrm{P}<0.05)$, and tumor growth in the combined groups was significantly slower than that of CG $(\mathrm{P}<0.05)$. The OS of rats was significantly different between the combined groups and control group $(\mathrm{P}<0.05)$, which were $19.9 \pm 3.21,31.2 \pm 6.48,27.1 \pm 5.59$, and $25.9 \pm 6.06$ days in groups $C G$, CG1, CG2 and CG3, respectively. Significant differences were observed between groups CG1 and CG3. The expression levels of VEGF in groups CG1, CG2 and CG3 were 45.6 $\pm 9.88,70.8 \pm 14.11$ and $75.3 \pm 9.82$, and were significantly lower than that in control groups $(85.8 \pm 11.26)$. The MVD in CG $(109.7 \pm 10.32)$ reached the peak value when compared to those in the three combined groups $(46.4 \pm 19.22,75.7 \pm 15.97$, and $90.5 \pm 12.71$, all $\mathrm{P}<0.05$ ). Furthermore, overexpression of VEGF and MVD showed significant positive correlation with poor OS.

Conclusions: These findings demonstrated that apatinib treatment enhanced anti-tumor effects of TAE via reducing tumor angiogenesis, suppressing tumor growth, and prolonging the OS of rats with liver tumors. Early administration of apatinib showed better therapeutic effects.

Keywords: Liver tumor; apatinib; vascular endothelial growth factor (VEGF); angiogenesis; administration sequences; transarterial embolization (TAE)

Submitted Sep 14, 2019. Accepted for publication Dec 10, 2019.

doi: $10.21037 /$ tcr.2019.12.97

View this article at: http://dx.doi.org/10.21037/tcr.2019.12.97 


\section{Introduction}

Liver cancer is one of the most common malignancies with bad prognosis, and it is also the second most lethal tumor after pancreatic cancer $(1,2)$. In 2017, there were approximately 854,000 new cases with liver cancer and 810,000 cases of deaths worldwide. More than half of the total number of cases and deaths were accounted in China alone (3). Transarterial embolization (TAE) and transarterial chemoembolization (TACE) are two widely used locoregional therapies for unresectable hepatocellular carcinoma (HCC) $(4,5)$ and are recommended as first-line therapies for patients with intermediate-stage HCC in the guidelines (3). TAE induces marked ischemic tumor necrosis by obstructing tumor-feeding arteries. Nevertheless, there are a significant number of HCC patients $(50-86 \%)$ with residual viable tumors (6), and tumor angiogenesis plays a great role in them.

Angiogenesis is the process by which new blood vessels develop from the existing vasculature in tumors for enhancing the supply of oxygen and nutrients to support tumor growth, and is regarded as one of the hallmarks in tumor malignant behaviors. Multiple evidences have indicated that vascular endothelial growth factor (VEGF) is the most important inducer during the process of angiogenesis $(7,8)$. Abnormal overexpression of VEGF secreted by tumor and stromal cells has profound consequences in tumor microenvironment, leading to solid tumorigenesis and invasion, and increasing the risks of metastatic dissemination and other malignant behaviors $(8,9)$. According to the previous studies, VEGF showed marked elevation in majority of HCC patients after TAE $(10,11)$, exerting its functions in angiogenesis and lymphangiogenesis, and acting as a conduit for malignant biological behavior via VEGFR-2-dependent signaling pathway (12-14). Therefore, there is a significant clinical application value of VEGF-2-blocking antibodies.

Apatinib can inhibit tumor neovascularization and play an anticancer role by effectively binding and inhibiting VEGF receptor 2 (VEGFR-2) $(15,16)$. Previous findings had proved the promising clinical efficacy of apatinib in advanced HCC (17). Also the therapeutic effects of apatinib combined with TACE showed encouraging outcomes in HCC patients (18-20). However, our preliminary experimental study had revealed differences in the administration time of targeted drug in rats with liver tumor after VEGF injection, demonstrating different antitumor therapeutic efficacies (21). Similarly, different studies have adopted different models and administration time of targeted drug in patients undergoing TACE, which led to controversies across these trials (22-24). Therefore, in this study, the optimal administration time among different treatment schedules for using apatinib combined with TAE was investigated.

\section{Methods}

\section{Materials}

Walker-256 tumor cells were purchased from the Laboratory Animal Center of Sun Yat-sen University. The experimental animals were also purchased from the Laboratory Animal Center of Sun Yat-sen University. Gelfoam was purchased from Jinling Pharmaceutical, Nanjing, China. Both rabbit anti-rat VEGF-165 polyclonal antibody and rabbit anti-rat CD34 polyclonal antibody were purchased from BOSTER, Inc, Wuhan, China.

\section{Establishment of xenograft tumor models}

All experiments in this study were carried out in accordance with the approved guidelines of Institutional Animal Care and Use Committee, Sun Yat-Sen University (No: SYSUIACUC-2018-000086). All animals were maintained in the Laboratory Animal Center of Sun Yat-sen University under specific pathogen-free conditions (SPF) and provided sterilized food and water.

Walker-256 tumor cells were thawed at $-80{ }^{\circ} \mathrm{C}$ and cultured as previously described (25). The establishment of walker-256 tumor-bearing donor rats was performed as previously described (21). Tumors were recognized after about 10 days, and subcutaneous tumors of approximately $1 \mathrm{~cm}$ in diameter were detected at the injection site on the lateral thigh.

In situ liver cancer after implantation of subcutaneous tumors was performed. The animals were acclimatized to the laboratory conditions for 1 week prior to the experiments. Next, the Sprague-Dawley (SD) rats (weighing 150-200 g) with subcutaneous tumors were obtained from a donor animal by mincing them into small cubes of about $1.0 \mathrm{~mm}^{3}$. The rats were then laparotomized by making a midline abdominal incision under intraperitoneal anesthesia with $3 \%$ pentobarbital sodium at a dose of $50 \mathrm{mg} / \mathrm{kg}$ under sterile conditions. In the abdominal cavity, the accessory left liver lobe was protruded laterally and a subcapsular tunnel was made by 22 -gauge short-beveled needles. Next, a fish- 
like fleshy tumor tissue cube was inserted into the tunnel via the needle and the wound was covered by a small piece of gelfoam in case of hemorrhage. The rats were then immediately returned to their home cages to recover after surgery and regain normal activity the next day.

\section{Experimental processing}

The xenograft tumor models were allowed to establish for 10 days, and then magnetic resonance imaging (MRI) (Magnetom Avanto 3.0 T; Siemens, Washington, DC) was performed to evaluate whether or not the liver tumor models were successfully established and to analyze the characteristics of liver tumor. The main scanning parameters were as follows: repetition time (TR) $1,500 \mathrm{~ms}$ and echo time (TE) $82.0 \mathrm{~ms}$ for $\mathrm{T}_{1} \mathrm{WI}$; TR $508.7 \mathrm{~ms}$ and TE $1.5 \mathrm{~ms}$ for $\mathrm{T}_{2} \mathrm{WI}$ (slice thickness $=3 \mathrm{~mm}$ ).

The xenograft tumor models of the aforementioned established 40 rats were randomly allocated into 4 groups in accordance with different administration methods of apatinib with 10 rats in each group. Control group (CG): TAE only; Combined Group 1 (CG1): apatinib was administered 3 days before TAE; Combined Group 2 (CG2): apatinib was administered simultaneously with TAE; and Combined Group 3 (CG3): apatinib was administered 3 days after TAE. TAE was performed by hepatic artery ligation (HAL), which was done by ligating the main branch of left hepatic artery. Apatinib was administered everyday for 10 days at a dose of $50 \mathrm{mg} / \mathrm{kg}$ by oral gavage (15).

\section{Imaging and pathological examination}

MRI was performed to analyze tumor growth and liver tumor characteristics 10 days after apatinib administration. The maximum diameter of liver tumor was calculated according to the RECIST criteria (26). The figure of tumor growth was drawn based on tumor size and time of implantation. Tumor tissues were excised from the rats after their natural death.

Immunohistochemical (IHC) examination was performed to evaluate the expression of both VEGF and CD34 in rat liver tumor biopsies. Tumor tissues were fixed in formalin, and embedded in paraffin by labeled streptavidin avidin biotin (LSAB) method. In brief, the tissue sections were dewaxed in xylene, rehydrated and then were dehydrated. After that, the tumor tissues were immersed in $10 \mathrm{mM}$ Tris and 0.5 MEDTA at $\mathrm{pH} 9.0$ and then were subjected to microwave twice for $15 \mathrm{~min}$. The sections were then undergoing incubation with $3 \% \mathrm{H}_{2} \mathrm{O}_{2}$ for 10 minutes to quench the activity of endogenous peroxidase. After that, the sections were incubated overnight at $4{ }^{\circ} \mathrm{C}$ with primary antibodies (dilutions: VEGF-165, 1: 40; CD34, 1: 40). The rabbit anti-rat VEGF-165 polyclonal antibodies and rabbit anti-rat CD34 polyclonal antibodies were used.

\section{Immunostaining}

Immunostaining was conducted independently and blindly by two experienced pathologists using light microscope (DM2500, Leica, Germany). The number of epithelial cells showing immunoreactivity for VEGF was determined by counting the brown-colored positive cells at $\times 200$ magnification in each slide, in which 5 fields were randomly selected to count the stained epithelial cells. The staining of endothelial cells for CD34 was performed to evaluate the microvascular density (MVD) and tumor angiogenesis. Five areas with the most prominent vascular density (hot spots) in each slice were selected at $\times 100$ power field and the average number of microvessel was calculated based on the number of vessel profiles in all the five 'hot spots' under $\times 200$ magnification. Any cell cluster or a microvessel with/without lumen showing positive CD34 staining was counted as a vessel, and this was performed according to the Weidner method (27).

\section{Statistical analysis}

All statistical analyses were performed using SPSS ver. 20.0 software. All values were expressed as means \pm SEM, and significant differences among the groups were analyzed by one-way ANOVA followed by LSD test. Survival analysis and curves were determined using Kaplan-Meier method, and overall survival (OS) among the groups was compared using log-rank test. Spearman rank correlation coefficients were utilized to analyze the correlations of OS and the expressions of VEGF and MVD. The difference was considered to be statistically significant if the error probability value was less than $0.05(\mathrm{P}<0.05)$.

\section{Results}

\section{Walker 256 liver tumors}

An MRI scan was performed to investigate whether the liver cancer models were successfully established and to determine the location and size of the tumor on the 10th 

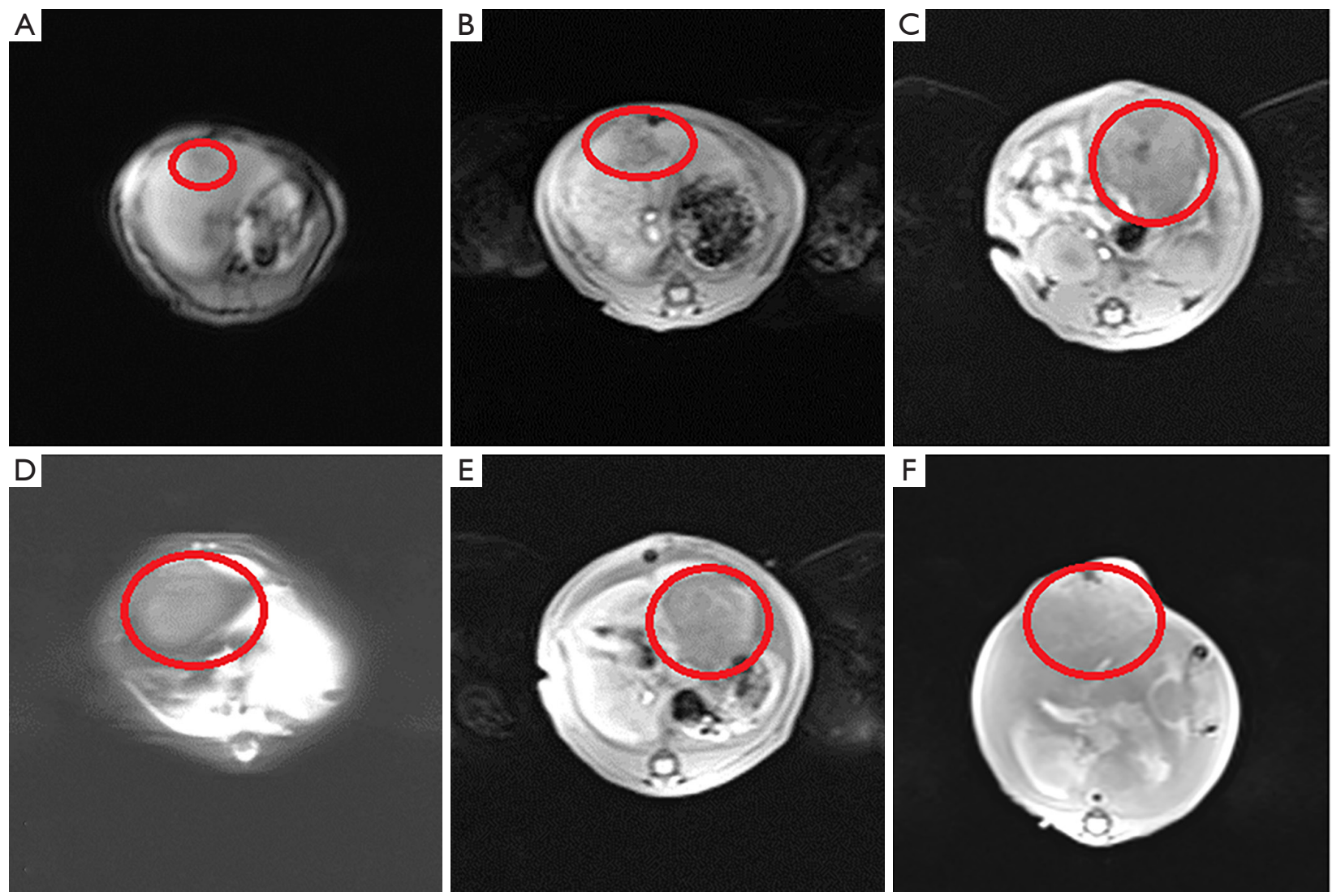

Figure 1 Representative MR images of liver cancer. (A) Rat liver lesion (red circle) on day 10 of tumor tissue implantation was slightly hypointense on T1WI; (B) the aforementioned lesion (red circle) was slightly hyperintense on T2WI; (C) T2WI MRI axial scans of rat liver cancer in control group 10 days after intervention; (D) T2WI MRI axial scans of rat liver cancer in apatinib administration 3 days pre-TAE group 10 days after intervention; (E) T2WI MRI axial scans of rat liver cancer in apatinib administration simultaneously with TAE group 10 days after intervention; (F) T2WI MRI axial scans of rat liver cancer in apatinib administration 3 days post-TAE group 10 days after intervention. MR, magnetic resonance.

day after inoculation of Walker-256 tumor cells. After that, the models with tumor diameter of $1.6-2.4 \mathrm{~cm}$ were selected as experimental animals and the size of liver tumor was taken as the baseline. The MRI with high decent quality showed localization of the nodular masses in the liver, with a slightly hypointense mass in $\mathrm{T}_{1} \mathrm{WI}$ and a slightly hyperintense mass in $\mathrm{T}_{2} \mathrm{WI}$ without obvious demarcation between the tumor and surrounding normal liver tissue (Figure $1 A, B)$. MR scan was again performed 10 days after the intervention, and the maximum diameter of the tumor was calculated according to the RECIST criteria (26). As shown by MR imaging, the size of intrahepatic tumors showed a significant increase when compared to the baseline $(\mathrm{P}<0.05)$, and the signal intensity was usually inhomogeneous and showed low intensity in the periphery and high signal intensity in the center with no uniformity in $\mathrm{T}_{2} \mathrm{WI}$ (Figure 1C,D,E,F). The size of the tumor in CG, CG1, CG2 and CG3 groups were $5.27 \pm 0.45,4.86 \pm 0.32$, $4.81 \pm 0.33$, and $4.83 \pm 0.31 \mathrm{~cm}$, respectively (Figure 2). The subjects in the groups showed significant differences to apatinib treatment when compared to the control, while no significant difference was observed between apatinib groups.

\section{Survival of animals in each experimental group}

The follow-up experiments were conducted in 10 animals of each group. As shown in Figure 3, rats treated with apatinib $(31.2 \pm 6.48,27.1 \pm 5.59$, and $25.9 \pm 6.06$ days in groups CG1, CG2 and CG3 group, respectively) had significantly longer life than those without apatinib treatment $(19.9 \pm 3.21$ days 
in control group) (Figure 3). The survival time was gradually decreased according to different administration schedules in CG1, CG2 and CG3 groups and showed significant differences between the group GC1 when compared to GC3 ( $\mathrm{P}=0.037)$. However, the results showed no significant differences between CG1 and CG2 (P=0.103) and CG2 and CG3 ( $\mathrm{P}=0.628)$ groups.

\section{Pathological findings}

The liver tumor specimens were excised to conduct HE staining and IHC examination after natural death. Histopathologic examination (Figure 4) results revealed that the tumor area showed signs of nonspecific inflammation with destruction of normal lobular architecture and the tumor atypical cells were irregular and arranged in sheets or nested pattern with round or oval nuclei and pale eosinophilic cytoplasm. The tumor cells demonstrated

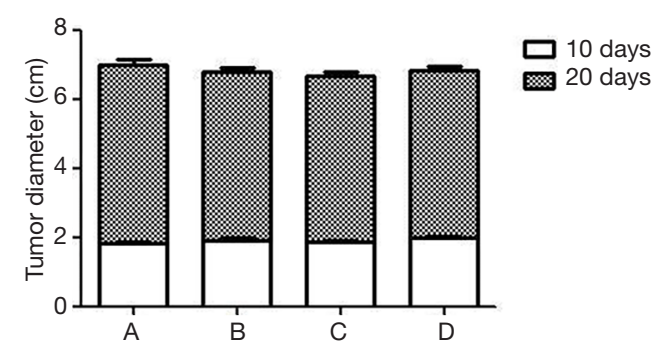

Figure 2 The average diameter of liver tumor at different timings. (A) Control group (CG); (B) combined Group 1 (CG1): apatinib administration 3 days before TAE; (C) combined Group 2 (CG2): apatinib administration simultaneously with TAE; (D) combined Group 3: apatinib administration 3 days after TAE control group. that the hyperchromatic nuclei were surrounded by scanty cytoplasm, which showed a pleomorphic and high mitotic activity with dual and even multi-core. There were no cellular structures in the area of eosinophilic necrosis. More importantly, large necrosis areas were observed in the tumor tissues of apatinib groups, and the number of tumor cells was significantly lower than that of the CG. As shown in Figure 4, early administration of apatinib before TAE resulted in larger range of tumor necrosis.

Studies showed that the VEGF expression was upregulated in liver tumors, and inhibition of VEGF function impaired tumor vascularization $(8,9)$. Herein, VEGF expression and MVD as determined by CD34 in liver tumors were studied (Figures 5,6). The VEGF immunoreactivity was localized in the cytoplasm of cancer cells, which was stained in brown color (Figure 5). As shown in Figures 5,7A, the number of VEGF-positive cells as detected by IHC are much more in CG $(85.8 \pm 11.26)$ when compared to apatinib groups $(45.6 \pm 9.88,70.8 \pm 14.11$, and $75.3 \pm 9.82$ in groups CG1, CG2 and CG3 group, respectively), showing statistically significant differences $(\mathrm{P}<0.05)$. The results showed that the expression of VEGF demonstrated a gradual decrease from group CG1 to group CG3 (Figure $7 A$ ), and significant difference occurred between group CG1 and groups CG2 and CG3 $(\mathrm{P}<0.05)$. Nevertheless, there was no significant difference in groups CG2 and CG3 $(\mathrm{P}=0.38)$ (Figure 5). Specific staining of capillary-like vessels by anti-CD34 was mainly observed as brown or dark-brown staining in the tumor tissues (Figure 6). The trend of MVD expression was similar to that of VEGF in the four groups, and the MVD expression was $109.7 \pm 10.32,46.4 \pm 19.22,75.7 \pm 15.97$, and $90.5 \pm 12.71$ in groups CG, CG1, CG2, and CG3, respectively, which
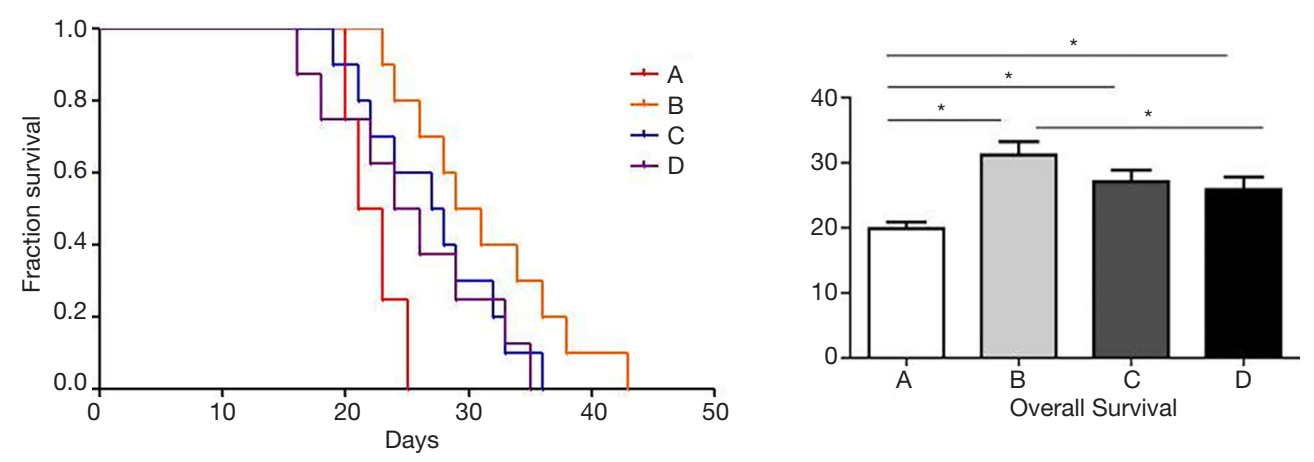

Figure 3 Survival curves and average survival time of rats in four groups. (A) Control group (CG); (B) combined Group 1 (CG1): apatinib administration 3 days before TAE; (C) combined Group 2 (CG2): apatinib administration simultaneously with TAE; (D) Combined Group 3 (CG3): apatinib administration 3 days after TAE control group ${ }^{*}, \mathrm{P}<0.05$ between the groups as shown by horizontal line). 

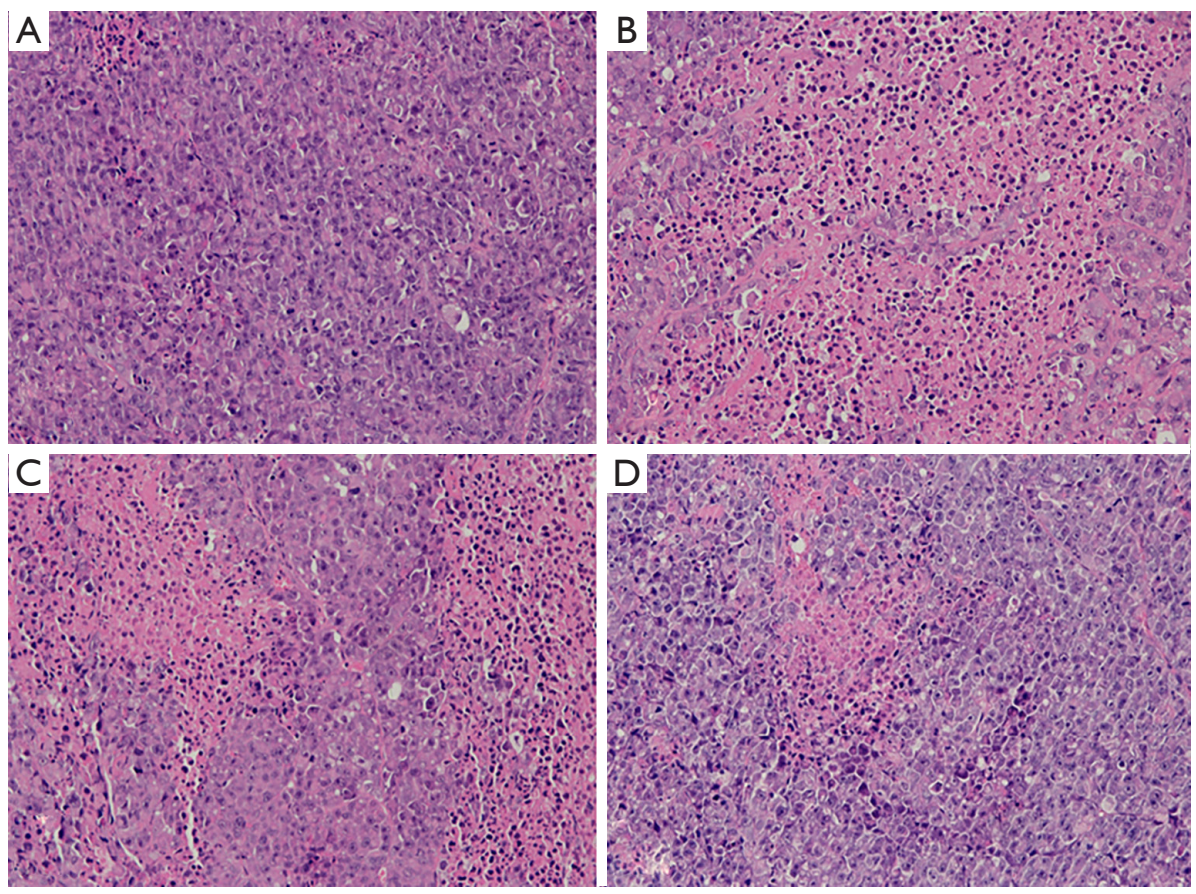

Figure 4 Representative HE imaging of liver cancer microscopically (×200). (A) Control group (CG); (B) combined Group 1 (CG1); (C) combined Group 2 (CG2); (D) combined Group 3 (CG3).
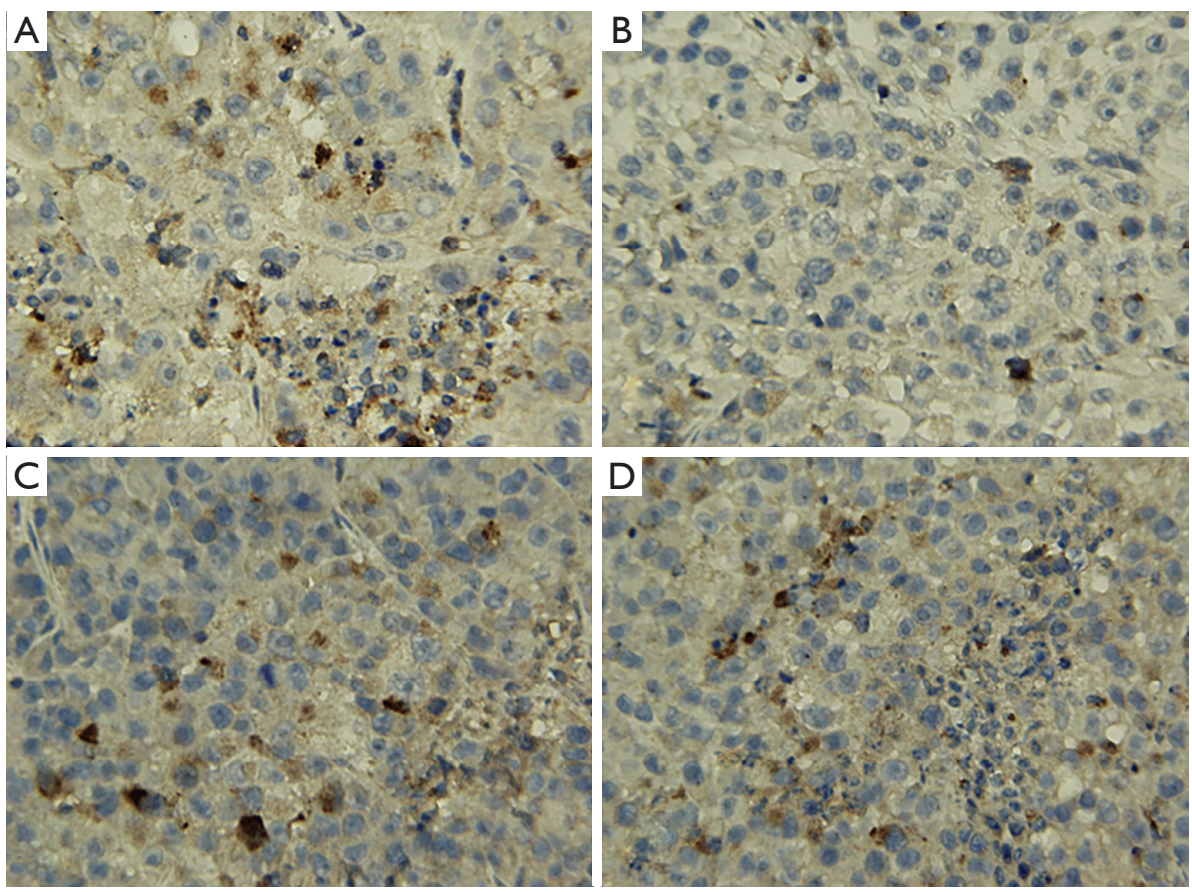

Figure 5 Immunohistochemical staining showed a positive VEGF reaction (×400). (A) Control group (CG); (B) combined Group 1 (CG1); (C) combined Group 2 (CG2); (D) combined Group 3 (CG3). 

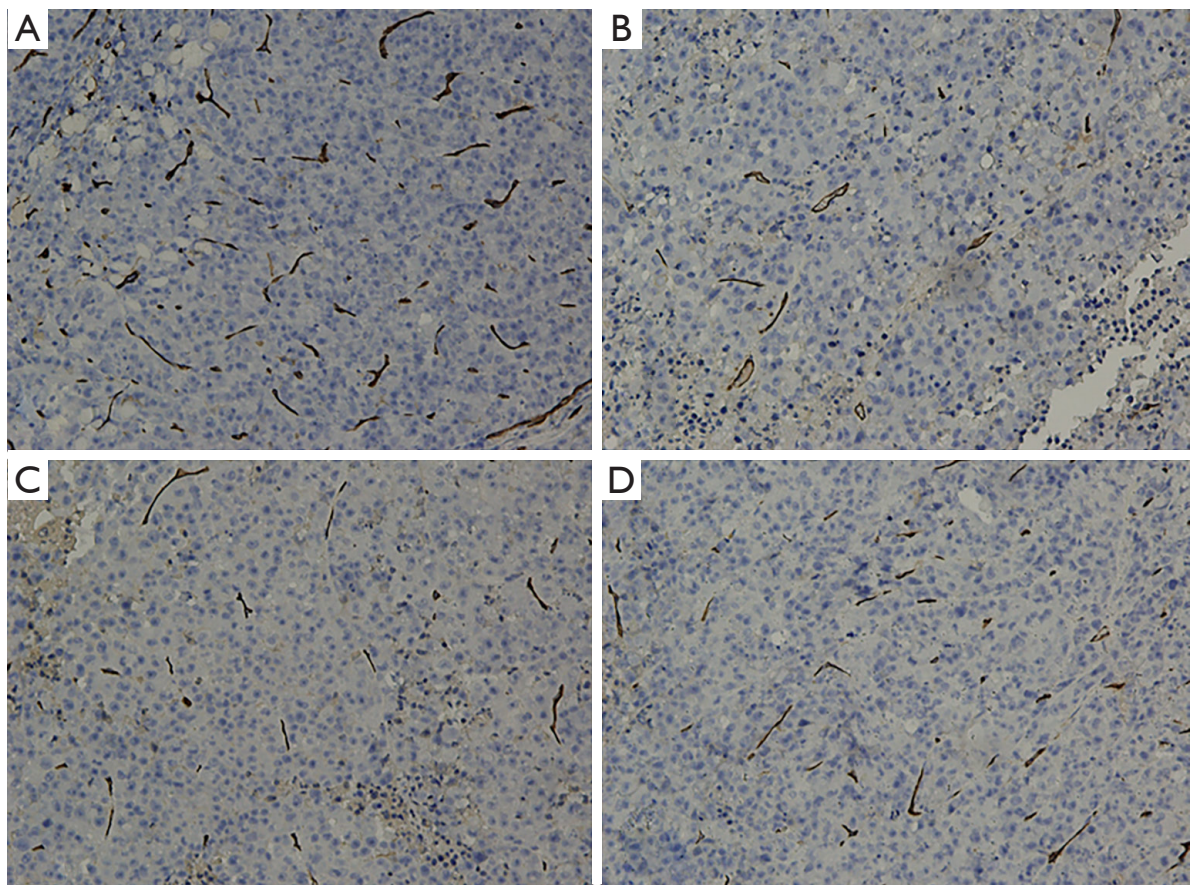

Figure 6 Immunohistochemical staining showed a positive CD34 reaction $(\times 200)$. Positive CD34 was identified by dark-brown staining. (A) Control group (CG); (B) combined Group 1 (CG1); (C) combined Group 2 (CG2); (D) combined Group 3 (CG3).
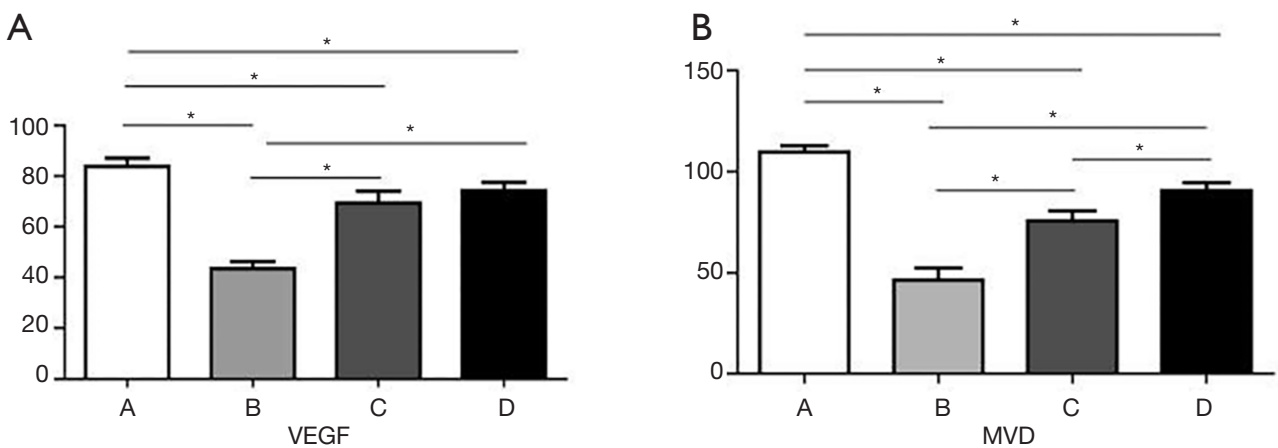

Figure 7 IHC analysis of expressions of VEGF and MVD in the four groups. (A) Control group (CG); (B) combined Group 1 (CG1); (C) combined Group 2 (CG2); (D) combined Group 3 (CG3) (*, P<0.05 between the two groups as shown by horizontal line).

showed statistically significant differences in any two groups $(\mathrm{P}<0.01)$ (Figure 7B).

\section{Correlation between tumor biological parameters}

VEGF and MVD are considered as important biomarkers for assessing the angiogenic activity of tumors and the results revealed an intense positive association. Our study (Figure 8) proved that the expression of VEGF showed a positive association with MVD and a positive correlation coefficient of 0.948 . A negative correlation was also found between OS and VEGF expression (correlational coefficient, -0.869). Similarly, the MVD and OS showed a negative correlation of -0.891 .

\section{Discussion}

HCC is a major public health problem with characteristics of high incidence, rapid progression and high mortality $(3,28-30)$. TACE and TAE were recommended as first- 

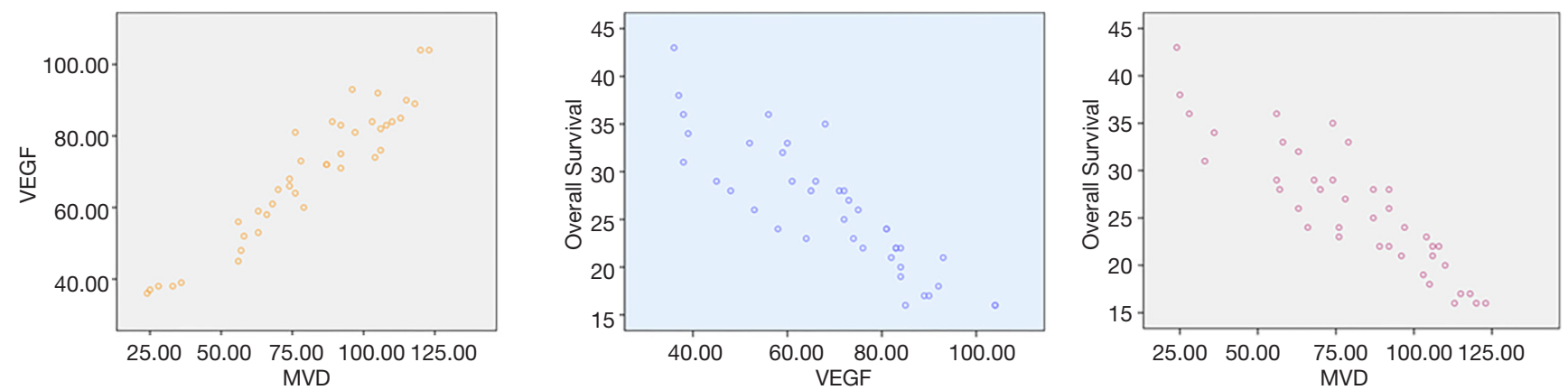

Figure 8 Correlation of OS and tumor angiogenesis. (A) The expression of VEGF was negatively correlated with MVD (R =0.948); (B) the expression of MVD was negatively correlated with OS (correlational coefficient, -0.869); (C) the correlation between MVD and OS was also negative at -0.891. MVD, microvascular density; OS, overall survival.

line treatment strategies, improving patient prognosis in patients with advanced HCCs (4,5,31-35). However, the survival benefits for patients undergoing these treatments are relatively modest (3), and the pitfalls of TAE in HCC included tumor residues and recurrence, in which aberrations in tumor angiogenesis played a key role (13). At present, VEGF is the strongest angiogenic factor in this process (36), and the expression of VEGF in remnant peripheral tumor tissues becomes higher, more invasive and metastatically active after TAE $(37,38)$. Therefore, inhibiting high expression levels of VEGF in tumor cells induced by TAE may become an important link to improve the long-term efficacy of TAE.

As a highly selective VEGFR-2 blocker, apatinib combined TACE was proved to be safe and improved the OS and progression-free survival in patients with HCC $(18,19)$. Although the therapy of targeted drugs including sorafenib combined with TACE had been proved effective and safe, the differences in the timings of anti-angiogenic treatment in patients undergoing TACE across these trials remain controversial $(24,39)$. Furthermore, the efficacy of differences in timings of apatinib administration combined with TACE still remained unknown. In the study, a liver cancer model using walker-256 tumor cells was established in SD rats. Furthermore, MRI was performed to efficiently monitor the progression of liver cancer and used to assess the subsequent therapeutic efficiency $(21,40)$. As shown in Figure 1, tumor size as shown demonstrated a significant increase when compared to the baseline $(2 \mathrm{~cm}$ in diameter with a spout protruding a further $20 \%$ ) according to the RECIST criteria (26). The signal intensity of the tumor in the groups post-treatment was inhomogeneous and showed low intensity in the periphery and high signal intensity in the center with no uniformity in $\mathrm{T}_{2} \mathrm{WI}$ (Figure 1C,D,E,F).
This meant that necrotic fractions were proportional to the area of the tumor. As expected, the differences in the size of tumors significantly decreased in groups subjected to apatinib when compared to CG, proving that apatinib could inhibit tumor growth in vivo. Similarly, studies were proved that apatinib can delay xenograft tumor growth in vivo (41). Yet no significant differences in the size of liver tumors occurred between any other combined therapy groups. Nonetheless, a difference in cell viability within tumor tissues was identified pathologically. As shown in Figure 4, large areas of eosinophilic necrosis without cellular structure were observed in the tumor tissues of combined groups, whereas little necrotic areas were observed in the CG (Figure 4A). Obviously, the range of tumor necrosis in early administration of apatinib was largest. As is well known, tumor necrosis is mainly due to lack of blood supply. Herein, tumor angiogenesis was studied by IHC staining. As shown in Figure 5, the VEGF expression showed a significantly lower in apatinib-treated groups when compared to the CG. And the expression of VEGF was gradually decreased from group CG1 to group CG3 (Figure $7 A$ ) with significant difference between group CG1 and groups CG2 and CG3. Furthermore, the trend of MVD expression was almost identical to that of VEGF in the four groups, and statistically differences were observed between any two groups. The OS of rats treated with apatinib combined with TAE was significantly longer than those without apatinib treatment, which was shown in Figure 3. Moreover, the OS of rats in groups CG1, CG2, and CG3 showed a decreasing trend based on different administration regimens with significant differences between CG1 and CG3. Nevertheless, no significant difference was observed in groups CG1 and CG2 $(\mathrm{P}=0.103)$ and groups CG2 and CG3 $(\mathrm{P}=0.628)$. Taken together, these 
results fully confirmed that early administration of apatinib demonstrated better outcomes. Several lines of evidence indicate that is angiogenesis was associated with tumor initiation and poor prognosis in HCC (42-44). Therefore, to analyze the relationship between MVD, VEGF, and OS, spearman rank correlation was performed. As shown in Figure 8, the expression of VEGF showed a strongly positive association with that of MVD, and there was a negative correlation between OS and VEGF expression (correlational coefficient, -0.869). Similarly, the correlation was also negative at -0.891 between MVD and OS.

\section{Conclusions}

In our study, liver xenograft tumor models were successfully established and their survival, tumor growth and the characteristics were analyzed via MRI. Apatinib treatment enhanced the anti-tumor effects of TAE via reducing tumor angiogenesis, suppressing tumor growth, and prolonging the OS of liver tumors of rats. Early administration of apatinib before TAE demonstrated better therapeutic effects.

\section{Acknowledgments}

Funding: None.

\section{Footnote}

Conflicts of Interest: The authors have completed the ICMJE uniform disclosure form (available at http://dx.doi. org/10.21037/tcr.2019.12.97). The authors have no conflicts of interest to declare.

Ethical Statement: The authors are accountable for all aspects of the work in ensuring that questions related to the accuracy or integrity of any part of the work are appropriately investigated and resolved. This study was approved by the Institutional Animal Care and Use Committee, Sun Yat-Sen University (SYSUIACUC-2018-000086).

Open Access Statement: This is an Open Access article distributed in accordance with the Creative Commons Attribution-NonCommercial-NoDerivs 4.0 International License (CC BY-NC-ND 4.0), which permits the noncommercial replication and distribution of the article with the strict proviso that no changes or edits are made and the original work is properly cited (including links to both the formal publication through the relevant DOI and the license). See: https://creativecommons.org/licenses/by-nc-nd/4.0/.

\section{References}

1. Villanueva A. Hepatocellular Carcinoma. N Engl J Med 2019;380:1450-62.

2. Jemal A, Ward EM, Johnson CJ, et al. Annual Report to the Nation on the Status of Cancer, 1975-2014, Featuring Survival. J Natl Cancer Inst 2017. doi: 10.1093/jnci/ djx030.

3. European Association for the Study of the Liver. EASL Clinical Practice Guidelines: Management of hepatocellular carcinoma. J Hepatol 2018;69:182-236.

4. Raoul JL, Sangro B, Forner A, et al. Evolving strategies for the management of intermediate-stage hepatocellular carcinoma: available evidence and expert opinion on the use of transarterial chemoembolization. Cancer Treat Rev 2011;37:212-20.

5. Park JW, Chen M, Colombo M, et al. Global patterns of hepatocellular carcinoma management from diagnosis to death: the BRIDGE Study. Liver Int 2015;35:2155-66.

6. Semenza GL. Targeting HIF-1 for cancer therapy. Nat Rev Cancer 2003;3:721-32.

7. Hashemi Goradel N, Ghiyami-Hour F, Jahangiri S, et al. Nanoparticles as new tools for inhibition of cancer angiogenesis. J Cell Physiol 2018;233:2902-10.

8. Viallard C, Larrivee B. Tumor angiogenesis and vascular normalization: alternative therapeutic targets. Angiogenesis 2017;20:409-26.

9. Li Y, Zhai Z, Liu D, et al. CD105 promotes hepatocarcinoma cell invasion and metastasis through VEGF. Tumour Biol 2015;36:737-45.

10. Li X, Feng GS, Zheng CS, et al. Expression of plasma vascular endothelial growth factor in patients with hepatocellular carcinoma and effect of transcatheter arterial chemoembolization therapy on plasma vascular endothelial growth factor level. World J Gastroenterol 2004;10:2878-82.

11. Wang $\mathrm{B}, \mathrm{Xu} \mathrm{H}, \mathrm{Gao} Z \mathrm{Z}$, et al. Increased expression of vascular endothelial growth factor in hepatocellular carcinoma after transcatheter arterial chemoembolization. Acta Radiol 2008;49:523-9.

12. Adams RH, Alitalo K. Molecular regulation of angiogenesis and lymphangiogenesis. Nat Rev Mol Cell Biol 2007;8:464-78.

13. Saharinen P, Eklund L, Pulkki K, et al. VEGF and 
angiopoietin signaling in tumor angiogenesis and metastasis. Trends Mol Med 2011;17:347-62.

14. Greenberg JI, Shields DJ, Barillas SG, et al. A role for VEGF as a negative regulator of pericyte function and vessel maturation. Nature 2008;456:809-13.

15. Tian S, Quan H, Xie C, et al. YN968D1 is a novel and selective inhibitor of vascular endothelial growth factor receptor-2 tyrosine kinase with potent activity in vitro and in vivo. Cancer Sci 2011;102:1374-80.

16. Liu J, Xu J, Zhang W, et al. Safety and Efficacy of DrugEluting Bead Transarterial Chemoembolization Combined with Apatinib in Patients with Advanced Hepatocellular Carcinoma. Acad Radiol 2019. [Epub ahead of print].

17. Yu WC, Zhang KZ, Chen SG, et al. Efficacy and Safety of apatinib in patients with intermediate/advanced hepatocellular carcinoma: A prospective observation study. Medicine (Baltimore) 2018;97:e9704.

18. Lu W, Jin XL, Yang C, et al. Comparison of efficacy between TACE combined with apatinib and TACE alone in the treatment of intermediate and advanced hepatocellular carcinoma: A single-center randomized controlled trial. Cancer Biol Ther 2017;18:433-8.

19. Chen S, Yu W, Zhang K, et al. Comparison of the efficacy and safety of Transarterial chemoembolization with and without Apatinib for the treatment of BCLC stage C hepatocellular carcinoma. BMC Cancer 2018;18:1131.

20. Fan W, Yuan G, Fan H, et al. Apatinib Combined With Transarterial Chemoembolization in Patients With Hepatocellular Carcinoma and Portal Vein Tumor Thrombus: A Multicenter Retrospective Study. Clin Ther 2019;41:1463-76.

21. Li N, Chen B, Lin R, et al. The earlier, the better: the effects of different administration timepoints of sorafenib in suppressing the carcinogenesis of VEGF in rats. Cancer Chemother Pharmacol 2018;81:207-16.

22. Huang Y, Chen B, Liu N, et al. Overall survival in response to sorafenib with transarterial chemoembolization for BCLC stage B hepatocellular carcinoma: propensity score analysis. Int J Clin Pharmacol Ther 2017;55:498-508.

23. Strebel BM, Dufour JF. Combined approach to hepatocellular carcinoma: a new treatment concept for nonresectable disease. Expert Rev Anticancer Ther 2008;8:1743-9.

24. Kudo M, Imanaka K, Chida N, et al. Phase III study of sorafenib after transarterial chemoembolisation in Japanese and Korean patients with unresectable hepatocellular carcinoma. Eur J Cancer 2011;47:2117-27.
25. Pigatto MC, Roman RM, Carrara L, et al.

Pharmacokinetic/pharmacodynamic modeling of etoposide tumor growth inhibitory effect in Walker-256 tumor-bearing rat model using free intratumoral drug concentrations. Eur J Pharm Sci 2017;97:70-8.

26. Schwartz LH, Seymour L, Litiere S, et al. RECIST 1.1 - Standardisation and disease-specific adaptations: Perspectives from the RECIST Working Group. Eur J Cancer 2016;62:138-45.

27. Weidner N, Semple JP, Welch WR, et al. Tumor angiogenesis and metastasis--correlation in invasive breast carcinoma. N Engl J Med 1991;324:1-8.

28. Connell LC, Harding JJ, Shia J, et al. Combined intrahepatic cholangiocarcinoma and hepatocellular carcinoma. Chin Clin Oncol 2016;5:66.

29. Siegel RL, Miller KD, Jemal A. Cancer statistics, 2016. Ca A Cancer Journal for Clinicians 2016;66:7.

30. Omata M, Cheng AL, Kokudo N, et al. Asia-Pacific clinical practice guidelines on the management of hepatocellular carcinoma: a 2017 update. Hepatol Int 2017;11:317-70.

31. Camma C, Schepis F, Orlando A, et al. Transarterial chemoembolization for unresectable hepatocellular carcinoma: meta-analysis of randomized controlled trials. Radiology 2002;224:47-54.

32. Llovet JM, Bruix J. Systematic review of randomized trials for unresectable hepatocellular carcinoma: Chemoembolization improves survival. Hepatology 2003;37:429-42.

33. Xu XL, Liu XD, Liang M, et al. Radiofrequency Ablation versus Hepatic Resection for Small Hepatocellular Carcinoma: Systematic Review of Randomized Controlled Trials with Meta-Analysis and Trial Sequential Analysis. Radiology 2018;287:461-72.

34. Llovet JM, Burroughs A, Bruix J. Hepatocellular carcinoma. Lancet 2003;362:1907-17.

35. Murata S, Mine T, Sugihara F, et al. Interventional treatment for unresectable hepatocellular carcinoma. World J Gastroenterol 2014;20:13453-65.

36. Chen J, Hou E. Progress of VEGF and its receptors in HCC angiogenesis and antiangiogenesis therapy. Modern Oncol 2016;24:498-502.

37. Ni Y, Ye X, Wan C, et al. Percutaneous microwave ablation (MWA) increased the serum levels of VEGF and MMP-9 in Stage I non-small cell lung cancer (NSCLC). Int J Hyperthermia 2017;33:435-9.

38. Mashreghi M, Azarpara H, Bazaz MR, et al. Angiogenesis biomarkers and their targeting ligands as potential targets 
for tumor angiogenesis. J Cell Physiol 2018;233:2949-65.

39. Pawlik TM, Reyes DK, Cosgrove D, et al. Phase II trial of sorafenib combined with concurrent transarterial chemoembolization with drug-eluting beads for hepatocellular carcinoma. J Clin Oncol 2011;29:3960-7.

40. Ayuso C, Rimola J, Vilana R, et al. Diagnosis and staging of hepatocellular carcinoma (HCC): current guidelines. Eur J Radiol 2018;101:72-81.

41. Peng S, Zhang $\mathrm{Y}$, Peng $\mathrm{H}$, et al. Intracellular autocrine VEGF signaling promotes EBDC cell proliferation, which can be inhibited by Apatinib. Cancer Lett 2016;373:193-202.

Cite this article as: Li N, Huang YH. The suppressing effects of VEGF-mediated angiogenesis at different administration sequences of apatinib and transarterial embolization in vivo. Transl Cancer Res 2020;9(2):1133-1143. doi: 10.21037/ tcr.2019.12.97
42. Buijs N, Oosterink JE, Jessup M, et al. A new key player in VEGF-dependent angiogenesis in human hepatocellular carcinoma: dimethylarginine dimethylaminohydrolase 1 . Angiogenesis 2017;20:557-65.

43. Matsuki M, Hoshi T, Yamamoto Y, et al. Lenvatinib inhibits angiogenesis and tumor fibroblast growth factor signaling pathways in human hepatocellular carcinoma models. Cancer Med 2018;7:2641-53.

44. Matsui D, Nagai H, Mukozu T, et al. VEGF in patients with advanced hepatocellular carcinoma receiving intraarterial chemotherapy. Anticancer Res 2015;35:2205-10. 